\title{
Electrochemotherapy in the Treatment of Cutaneous Metastases from Breast Cancer: A Multicenter Cohort Analysis
}

\author{
C. Cabula, MD', L. G. Campana, $\mathrm{MD}^{2}$, G. Grilz, $\mathrm{MD}^{3}$, S. Galuppo, $\mathrm{MD}^{2}$, R. Bussone, $\mathrm{MD}^{3}$, L. De Meo, $\mathrm{MD}^{4}$, \\ A. Bonadies, $\mathrm{MD}^{5}$, P. Curatolo, $\mathrm{MD}^{6}$, M. De Laurentiis, $\mathrm{MD}^{7}$, M. Renne, $\mathrm{MD}^{8}$, S. Valpione, $\mathrm{MD}^{2}$, T. Fabrizio, $\mathrm{MD}^{9}$, \\ N. Solari, $\mathrm{MD}^{10}$, M. Guida, $\mathrm{MD}^{11}$, A. Santoriello, $\mathrm{MD}^{12}$, M. D'Aiuto, $\mathrm{MD}^{13}$, and R. Agresti, $\mathrm{MD}^{14}$ \\ ${ }^{1}$ Oncologic Surgery, Ospedale Oncologico A. Businco, Cagliari, Italy; ${ }^{2}$ Veneto Institute of Oncology IOV-IRCCS, Padua, \\ Italy; ${ }^{3}$ Breast Surgery Unit, Ospedale Le Molinette, Turin, Italy; ${ }^{4}$ Humanitas-Centro Catanese di Oncologia, Catania, Italy; \\ ${ }^{5}$ Plastic Surgery Unit, San Gallicano Dermatologic Institute, Rome, Italy; ${ }^{6}$ Dermatology and Plastic Surgery Department, \\ La Sapienza University, Rome, Italy; ${ }^{7}$ Seconda Università di Napoli, Naples, Italy; ${ }^{8}$ Fondazione T. Campanella, Catanzaro, \\ Italy; ${ }^{9}$ Plastic Surgery Unit, IRCCS, Referral Cancer Center of Basilicata, Rionero in Vulture, Italy; ${ }^{10}$ Surgical Unit 1 , \\ IRCCS San Martino-IST, Genoa, Italy; ${ }^{11}$ Medical Oncology Unit, Istituto dei Tumori, Bari, Italy; ${ }^{12}$ Department of \\ Medicine and Surgery, "Federico II" University, Naples, Italy; ${ }^{13}$ Breast Surgery Unit, Istituto Nazionale Tumori \\ "Pascale", Naples, Italy; ${ }^{14}$ Breast Surgery Unit, Fondazione IRCCS Istituto Nazionale dei Tumori, Milan, Italy
}

\begin{abstract}
Background. The management of breast cancer (BC) skin metastases represents a therapeutic challenge. Electrochemotherapy (ECT) combines the administration of bleomycin with temporary permeabilization induced by locally administered electric pulses. Preliminary experience with $\mathrm{ECT}$ in $\mathrm{BC}$ patients is encouraging.

Methods. A total of 125 patients with BC skin metastases who underwent ECT between 2010 and 2013 were enrolled onto a multicenter retrospective cohort study. The treatment was administered following the European Standard Operative Procedures of Electrochemotherapy. Tumor response was clinically assessed adapting the Response Evaluation Criteria in Solid Tumors, and toxicity was evaluated according to Common Terminology Criteria for Adverse Events 4.0. Cox regression analysis was used to identify predictive factors.
\end{abstract}

Results. Response was evaluable in 113 patients for 214 tumors (median 1 per patient, range 1-3). The overall

C. Cabula and L. G. Campana have contributed equally to this article, and both should be considered first author.

(C) The Author(s) 2015. This article is published with open access at Springerlink.com

First Received: 13 May 2015;

Published Online: 5 August 2015

R. Agresti, MD

e-mail: roberto.agresti@istitutotumori.mi.it response rate after 2 months was $90.2 \%$, while the complete response $(\mathrm{CR})$ rate was $58.4 \%$. In multivariate analysis, small tumor size $(P<0.001)$, absence of visceral metastases $(P=0.001)$, estrogen receptor positivity $(P=0.016)$, and low Ki-67 index $(P=0.024)$ were significantly associated with CR. In the first $48 \mathrm{~h}, 10.4 \%$ of patients reported severe skin pain. Dermatologic toxicity included grade 3 skin ulceration $(8.0 \%)$ and grade 2 skin hyperpigmentation $(8.8 \%)$. Tumor 1-year local progression-free survival was $86.2 \%$ (95\% confidence interval 79.3-93.8) and $96.4 \%$ (95\% confidence interval 91.6100 ) in the subgroup of those with CR.

Conclusions. In this study, small tumor size, absence of visceral metastases, estrogen receptor positivity, and low Ki67 index were predictors of CR after ECT. Patients who experienced CR had durable local control. ECT represents a valuable skin-directed therapy for selected patients with BC.

Skin metastases from breast cancer (BC) are often symptomatic for ulceration, bleeding, and pain, and they may represent a challenge for clinicians, particularly in heavily pretreated patients. Surgical resection, radiotherapy, and systemic therapies can be variously combined according to individual patient characteristics, tumor features, and physician choice. ${ }^{1}$ When surgical excision is not possible, radiotherapy ensures sustained local control, even if this is not feasible in preirradiated areas. ${ }^{2}$ Systemic therapies, such as endocrine treatment, chemotherapy, and 
targeted agents, represent valuable options, depending on the molecular subtype of $\mathrm{BC}$ and prior therapies. ${ }^{3}$ Application of topic chemotherapy and laser ablation is limited to cancers confined to the top layer of skin. ${ }^{4}$ Electrochemotherapy (ECT) combines the administration of a poorly permeant cytotoxic agent, such as bleomycin (BLM), with the local application of electric pulses that induce reversible electroporation, thus improving drug diffusion into cells. ${ }^{5}$ ECT was introduced in 2006, demonstrating a high rate of efficacy and favorable toxicity profile in a European multicenter study on skin metastases from different tumor histotypes. ${ }^{6}$ In this study, the objective response (OR) rate on treated tumor nodules was $89.0 \%$ with complete regression in $73.3 \%$ of cases. A recently published meta-analysis including 47 prospective studies comparing five skin-directed therapies (ECT, radiation, photodynamic therapy, intralesional therapy, and topical therapy), ECT demonstrated an OR rate of $75.4 \%$ (CR rate, $47.5 \%$ ) with a low toxicity profile (grade 3 in less than $6 \%$ of patients). ${ }^{7}$ In this analysis, melanoma and BC comprised $96.8 \%$ of all cutaneous metastases, with similar response rates.

To our knowledge, published data on ECT in BC patients with cutaneous skin metastases are based on small, single-center, heterogeneous series. Consequently, these series do not allow for identification of clinical and/or biologic factors that are reliably predictive of ECT response. ${ }^{8}$ The aim of our study was to provide a systemic analysis on a large series of BC patients treated with ECT, evaluating potential predictive factors of response to treatment.

\section{PATIENTS AND METHODS}

\section{Patients}

Between January 2010 and June 2013, the Italian Senological Group for Electrochemotherapy (GISEL), involving 13 Italian institutions, performed this multicenter retrospective cohort study. Inclusion criteria for ECT included BC patients with cutaneous and/or subcutaneous histologically confirmed metastases. Exclusion criteria for ECT included tumors in close proximity to a cardiac pacemaker; allergy to BLM; prior cumulative dose of BLM exceeding 250,000 IU/m ${ }^{2}$; serum creatinine $>150 \mu \mathrm{mol} / \mathrm{L}$; lung fibrosis; and pregnancy or lactation. Patients were enrolled regardless of the presence of other metastases. The respective institutional review boards of the participating institutions approved the study. All patients gave informed consent for the procedure and for utilization of their data for scientific purposes. Clinical records were anonymously entered into a dedicated encrypted online database.
Evaluation of Estrogen Receptor (ER), Progesterone Receptor (PgR), HER2 Status, and Ki-67 Index

Histologic diagnosis, immunohistochemical analysis, and fluorescence in situ hybridization for HER2 gene amplification (in case of inconclusive results on HER2 status) were performed according to international guidelines. The cutoff for ER and PgR positivity was $1 \%$ of cells with positive nuclear staining. ${ }^{9}$ Positivity for HER2 was determined by either immunohistochemistry $3+$ or fluorescence in situ hybridization amplification. The cutoff point for the Ki-67 labeling index was $14 \% .{ }^{10}$ Surrogate subtypes were defined according to the criteria established by the St. Gallen International Breast Cancer Conference. ${ }^{11}$

\section{Treatment}

The European Standard Operative Procedures of Electrochemotherapy (ESOPE) were used for all patients. ${ }^{12}$ Accordingly, the dose and route of BLM administration were adapted to the number and size of tumors in case of intratumoral injection, and to the patient's body surface area in case of intravenous infusion. The procedure was scheduled in a day-hospital regimen, and patients were usually discharged after an observation period of $24 \mathrm{~h}$.

\section{Patient Assessment}

Patients were evaluated after 1 and 2 weeks for acute toxicity and at 4 and 8 weeks for late toxicity and tumor response; subsequent follow-up visits were planned every 3-4 months. Among 125 patients, 12 (9.6\%) were followed for less than 2 months after ECT and were not considered for assessment of response.

For each patient, up to a maximum of five measurable tumors were registered as target lesions. The sum of their maximum diameters represented the baseline measurement for assessment of tumor response, which was clinically performed by Response Evaluation Criteria in Solid Tumors $1.1 .^{13}$ In case of the presence of many confluent nodules, when it was impossible to count their exact number, they were considered as a single entity and measured as a single area of treatment. Treatment toxicity and adverse events were graded according to the Common Terminology Criteria for Adverse Events $4 .{ }^{14}$ Pain was graded according to a $0-10$ numeric pain intensity scale $(0=$ no pain, $10=$ maximum pain $) .{ }^{15}$

\section{Statistical Analysis}

In descriptive analyses, continuous variables are reported as median value and interquartile range and categorical variables are reported as absolute number and percentage. 
Evaluation of tumor response was performed by contingency tables and Pearson's $\chi^{2}$ test.

Survival curves were estimated by the Kaplan-Meier method and compared to the log rank test. Hazard ratios were calculated by a Cox proportional risk model, after proportional hazard assumption confirmation with Schoenfeld residuals. Local progression-free survival (LPFS) was calculated from achievement of response in the treated area to local progression of disease, including the appearance of new nodules in the same area, or last followup. Statistical analyses were performed by SPSS 22.0 (IBM) software.

\section{RESULTS}

\section{Patient and Disease Characteristics}

Baseline patient and disease characteristics are reported in Table 1. The prevalent tumor histotype was infiltrating ductal carcinoma (76.6\%). Tumor, node, metastasis classification of primary BC was $\mathrm{T} 1-\mathrm{T} 2$ in $48 \%$ patients and T3-T4 in 52\%; $67.2 \%$ patients had lymph node involvement, and $22.4 \%$ had distant metastases.

The median number of target lesions was 1 (range 1-3), with a median size of $21 \mathrm{~mm}$ (range $15-45 \mathrm{~mm}$ ). The overwhelming majority of lesions-222 (92.9\%) of 239were localized on the chest wall.

Forty-one patients $(32.8 \%)$ received chemotherapy in a neoadjuvant setting at the time of primary $\mathrm{BC}$, while 62 patients $(49.6 \%)$ underwent chemotherapy in an adjuvant setting. Seventy-one patients $(56.8 \%)$ received adjuvant endocrine treatment. All patients had received at least one previous systemic treatment for metastatic disease. Specifically, 39 patients $(31.2 \%)$ received chemotherapy (median of two lines of treatment, range 1-6) and 69 patients received endocrine therapy (median two lines of treatment, range 13). Fifty-three patients (42.4\%) underwent adjuvant radiotherapy and 15 patients $(12.0 \%)$ were irradiated for the presence of skin metastases. As a result, 92 (38.5\%) of 239 target lesions in the present study were located in preirradiated skin. There were more previous systemic treatments in patients with triple negative (median 3, range 1-7) and HER2 positive (median 3, range 2-6) $\mathrm{BC}$ than in patients with luminal A-like (median 1, range 0-6), luminal B-like (median 2, range 0-6), and luminal B-like, HER2-positive tumors (median 2 , range $0-5, P=0.042$ ).

\section{Treatment}

In $92(73.6 \%)$ of 125 patients, ECT was administered under general anesthesia or sedation, while local anesthesia was used in the remaining 33 patients $(26.4 \%)$. BLM was
TABLE 1 Patient characteristics $(n=125)$

\begin{tabular}{|c|c|}
\hline Characteristic & $\begin{array}{l}\text { Median (range) } \\
\text { or } n(\%)\end{array}$ \\
\hline Age (years) & $63(54-72)$ \\
\hline \multicolumn{2}{|l|}{ Histology } \\
\hline IDC & $97(76.6)$ \\
\hline Non-IDC & $28(23.4)$ \\
\hline Time since occurrence of skin metastases (mo) & $32(9-109)$ \\
\hline \multicolumn{2}{|l|}{ Skin metastases $(n=239)$} \\
\hline No. per patient & $1(1-3)$ \\
\hline Size (mm) & $21(15-45)$ \\
\hline \multicolumn{2}{|l|}{ Location } \\
\hline Chest & $222(92.9)$ \\
\hline Other site & $17(7.1)$ \\
\hline \multicolumn{2}{|l|}{ Skin condition } \\
\hline Previous radiotherapy & $92(38.5)$ \\
\hline Lymphedema & $30(12.6)$ \\
\hline Ulceration & $64(26.8)$ \\
\hline \multicolumn{2}{|l|}{ Immunohistochemistry } \\
\hline ER positive & $72(57.6)$ \\
\hline PgR positive & $72(57.6)$ \\
\hline HER2 overexpression & $35(28.0)$ \\
\hline Ki-67<14\% & $63(50.4)$ \\
\hline \multicolumn{2}{|l|}{ Surrogate subtypes ${ }^{\mathrm{a}}$} \\
\hline Luminal A-like & $23(18.4)$ \\
\hline Luminal B-like (HER2 negative) & $22(17.6)$ \\
\hline Luminal B-like (HER2 positive) & $18(14.4)$ \\
\hline Triple negative & $35(28)$ \\
\hline HER2 & $11(8.8)$ \\
\hline \multicolumn{2}{|l|}{ Previous treatments ${ }^{\mathrm{b}}$} \\
\hline Radiotherapy & $68(54.4)$ \\
\hline Chemotherapy & $92(73.6)$ \\
\hline Endocrine therapy & $71(56.8)$ \\
\hline Targeted therapy & $14(11.2)$ \\
\hline Surgery for skin metastases & $89(71.2)$ \\
\hline
\end{tabular}

$I D C$ invasive ductal carcinoma, $E R$ estrogen receptor, $P R$ progesterone receptor

${ }^{\text {a }}$ Assessed on 113 patients, according to St. Gallen consensus ${ }^{11}$

b Any setting

administered intravenously in 100 patients $(80.0 \%)$ and intratumorally in 25 (20.0\%). Of 239 tumors, 207 $(86.6 \%)$ were electroporated with a hexagonal-array needle electrode, $10(4.2 \%)$ with a linear-row needle electrode, $13(5.4 \%)$ with a plate electrode, and $9(3.8 \%)$ with multiple electrode types.

\section{Toxicity}

No serious adverse events were reported during the procedure. 
TABLE 2 Toxicity within 2 months after electrochemotherapy $(n=125)$

\begin{tabular}{|c|c|c|c|c|}
\hline Toxicity & Any grade, $n(\%)$ & Grade $1, n(\%)$ & Grade $2, n(\%)$ & Grade $3, n(\%)$ \\
\hline Skin pain & $79(63.2)$ & $28(22.4)$ & $38(30.4)$ & $13(10.4)$ \\
\hline Skin ulceration & $41(32.8)$ & 17 (13.6) & $14(11.2)$ & $10(8.0)$ \\
\hline Skin hyperpigmentation & $34(27.2)$ & $23(18.4)$ & $11(8.8)$ & - \\
\hline Body odor & $10(8)$ & $4(3.2)$ & $6(4.8)$ & - \\
\hline Nausea & $10(8)$ & $10(8)$ & $0(0)$ & $0(0)$ \\
\hline Skin infection & $9(7.2)$ & $6(4.8)$ & $2(1.6)$ & $1(0.8)$ \\
\hline Flulike symptoms & $8(6.4)$ & $8(6.4)$ & $0(0)$ & $0(0)$ \\
\hline Fever & $7(5.6)$ & $7(5.6)$ & $0(0)$ & $0(0)$ \\
\hline Rash & $5(4)$ & $1(0.8)$ & $4(3.2)$ & $0(0)$ \\
\hline Soft tissue infection & $2(1.6)$ & $0(0)$ & $2(1.6)$ & $0(0)$ \\
\hline Vomiting & $2(1.6)$ & $2(1.6)$ & $0(0)$ & $0(0)$ \\
\hline Localized edema & $1(0.8)$ & $0(0)$ & $1(0.8)$ & $0(0)$ \\
\hline Postoperative hemorrhage & $1(0.8)$ & $0(0)$ & $1(0.8)$ & $0(0)$ \\
\hline
\end{tabular}

Toxicity data reported within the first 2 months are presented in Table 2. Paracetamol and nonsteroidal antiinflammatory agents were effective in controlling postprocedural pain in all but four patients, who required narcotics, although as a single administration. The incidence of skin ulceration did not differ significantly depending on previous radiation $(41.3 \%$ of previous skin radiations vs. $30 \%$ no previous skin radiation, $P=0.436$ ). After the first ECT, 96 patients were asked if they would agree to receive another course of treatment, if required, and $96.9 \%$ declared that they were potentially favorable.

\section{Tumor Response}

Among 125 patients, the follow-up of $12(9.6 \%)$ was less than 2 months after ECT; these subjects were not evaluated for response. Therefore, $113(90.4 \%)$ of 125 patients and $214(89.5 \%)$ of 239 target lesions were evaluated for tumor response. Two months after ECT, per-tumor response was as follows: CR $68.5 \%$, partial response (PR) $23.5 \%$, stable disease $6.6 \%$, progressive disease $0.9 \%$, and not evaluable $0.5 \%$ as a result of inflammatory reaction and crust formation. Accordingly, the OR rate was $92 \%$ (Fig. 1).

Sixty-six patients (58.4\%) experienced CR, 36 $(31.8 \%)$ PR, $8(7.1 \%)$ stable disease, and $2(1.8 \%)$ had progressive disease; in 1 patient $(0.9 \%)$, tumor response was not evaluable as a result of local skin conditions. Overall, the per-patient OR rate was $90.2 \%$.

The variables associated with response are shown in Table 3. The CR rate was higher in small $(<3 \mathrm{~cm})$ rather than large $(\geq 3 \mathrm{~cm})$ tumors (80.3 vs. $46.1 \%, P<0.0001)$ and in patients without visceral metastases rather than in those with visceral involvement $(80.5$ vs. $55.0 \%$, $P<0.001)$. The $\mathrm{CR}$ rate was also higher among ER-positive (77.2 vs. $59.8 \%$ in ER-negative, $P=0.006$ ) and low proliferating tumors $(\mathrm{Ki}-67<14 \%, 79.5 \%$ vs. $\mathrm{Ki}$ $67>14 \%, 58.8 \% ; P<0.001)$. In multivariate analysis, tumor size $<3 \mathrm{~cm}$ was confirmed to be the most powerful predictor of $\mathrm{CR}(P<0.001)$, followed by the absence of visceral metastases $(P=0.001), \quad$ ER-positive status $(P=0.016)$, and low Ki-67 $(P=0.024)$.

The distribution of tumor response according to the $\mathrm{BC}$ intrinsic subtypes is presented in Table 4 . The CR rate in patients with luminal A-like disease was significantly higher compared to all other subgroups (73.9 vs. $54.7 \%$, $P=0.02)$. There was no significant difference in tumor size among $\mathrm{BC}$ subtypes $(P=0.262)$.

There was no significant association between response and several clinical (patient age, $P=1.00$; type of surgery on primary $\mathrm{BC}, P=0.070$; time from primary $\mathrm{BC}$ to recurrence, $P=0.269$; presence of lymphedema, $P=0.636$; previous radiation, $P=1.00$ ) and procedural (anesthesiology technique, $P=0.377$; electrode type, $P=0.799$; route of BLM administration, $P=0.606$; number of electric pulses, $P=0.842$ ) parameters.

\section{Local Tumor Control}

Median follow-up time was 5.9 months (range 358 months). Median LPFS was not reached. One-year LPFS was $86.2 \%$ (95\% confidence interval [CI] 79.393.8) (Fig. 2).

One-year LPFS in patients who experienced a CR was $96.4 \%$ (95 \% CI 91.6-100). In multiple Cox regression analysis, tumor size was the only significant prognostic factor for LPFS (Table 3).

One-year LPFS survival in patients with small $(<3 \mathrm{~cm})$ tumors was $97.4 \%$ (95\% CI 92.6-100), whereas in those with larger tumors $(\geq 3 \mathrm{~cm}$ ), it was $75.6 \%$ (95\% CI 63.9$83.4 P=0.005)$. 

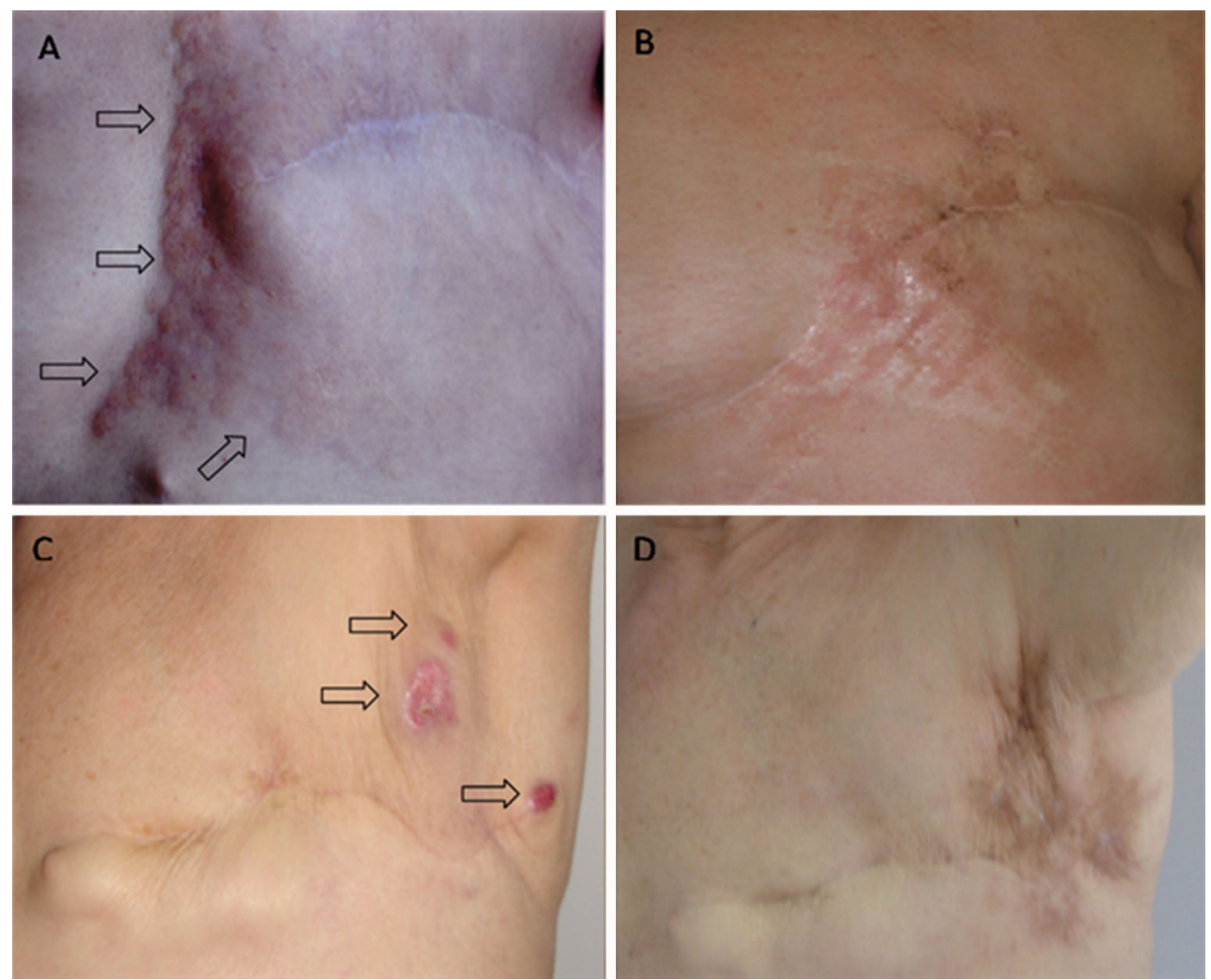

FIG. 1 Skin metastases from BC treated with ECT in two patients. Baseline presentation (a, c) and 1-year follow-up (b, d). Arrows contour tumor spread or indicate skin metastases

\section{DISCUSSION}

This study showed for the first time that a subgroup of BC patients, identified by routinely used immunohistochemical markers, was particularly sensitive to ECT with BLM. To our knowledge, this cohort analysis was based on the largest series of BC patients treated by ECT to date.

ECT was mainly administered under general sedation or general anesthesia (74\% of patients), while the preferential route for BLM administration was intravenous infusion (80\% of patients). In most cases (89\%), treated tumors were managed using a hexagonal-array, $20 \mathrm{~mm}$ long needle electrode. The most frequently reported adverse effects were transient pain and dermatologic toxicity. ${ }^{16-22}$ Generally, treated skin develops a transient inflammatory reaction. Occasionally, erosions or ulcerations may occur, followed by crust formation. In case of tumor regression, the skin may appear slightly less pigmented, while in some patients it is possible to observe local skin hyperpigmentation, which is a well-known effect of BLM. In patients with locally advanced disease, tumor shrinkage after ECT may cause tissue ulceration requiring specialist wound care. ${ }^{22}$ Nevertheless, previous experience has demonstrated that effective management of cutaneous metastases provided symptomatic relief and better quality of life to patients. ${ }^{20}$

With the present study, we confirm the absence of systemic adverse effects of ECT, as well as a favorable toxicity profile (grade 3 ulceration in $8 \%$ of patients, according to the meta-analysis of Spratt et al., grade 2 hyperpigmentation in $8.8 \%$ ), and a high level of acceptance. $^{7}$ Patients experienced minimal discomfort and needed small amounts of postprocedural analgesics; further, only $10 \%$ of adverse effects were severe, with the exception of transient pain within the first $48 \mathrm{~h}$. As a result, $97 \%$ of the 96 patients who were asked if they would agree to receive further treatment responded favorably. Our results are in line with the ESOPE study, where more than $90 \%$ of patients declared that they were potentially amenable to treatment. ${ }^{6}$

Melanoma and BC represented more than $95 \%$ of tumors included in two recently published meta-analyses where the indicated $\mathrm{CR}$ rates after ECT were 59 and $57.5 \%$, respectively. ${ }^{7,23}$ In the present study, the OR rate was $90.2 \%$, with a CR rate of $58.4 \%$, in agreement with the ESOPE study which reported an OR rate of $90.4 \%$, with $64.3 \%$ of patients experiencing $\mathrm{CR}^{6}{ }^{6}$ A recently published clinical trial on 55 patients, representing the 


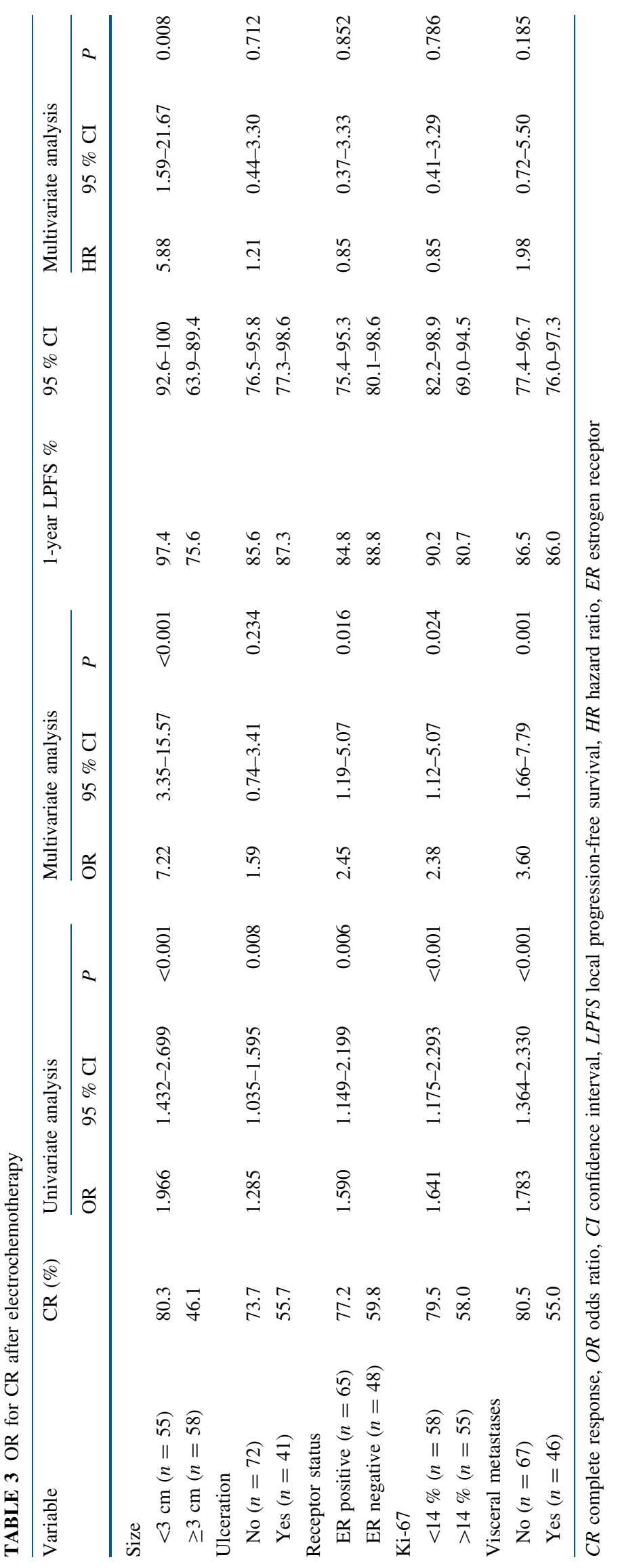


TABLE 4 Tumor response to electrochemotherapy according to surrogate definition of breast cancer intrinsic subtypes

\begin{tabular}{llllll}
\hline Response & $\begin{array}{l}\text { Luminal A-like } \\
(n=23), n(\%)\end{array}$ & $\begin{array}{l}\text { Luminal B-like }(H E R 2 \\
\text { negative })(n=22), n(\%)\end{array}$ & $\begin{array}{l}\text { Luminal B-like }(H E R 2 \\
\text { positive })(n=18), n(\%)\end{array}$ & $\begin{array}{l}\text { Triple negative } \\
(n=35), n(\%)\end{array}$ & $\begin{array}{l}\text { HER2 positive } \\
(n=11), n(\%)\end{array}$ \\
\hline CR & $17(73.9)$ & $11(50.0)$ & $10(55.6)$ & $20(57.1)$ & $6(54.5)$ \\
PR & $4(17.4)$ & $9(40.9)$ & $5(27.8)$ & $11(31.4)$ & $5(45.5)$ \\
SD & $1(4.3)$ & $2(9.1)$ & $1(5.6)$ & $4(11.4)$ & $0(0)$ \\
PD & $1(4.3)$ & $0(0)$ & $1(5.6)$ & $0(0)$ & $0(0)$ \\
NA & $0(0)$ & $0(0)$ & $1(5.6)$ & $0(0)$ & $0(0)$
\end{tabular}

According to Goldhirsh et al. ${ }^{11} ; n=109$ (in four patients, there was no reliable pathologic information). Luminal A-like tumors (ER and PgR positive, HER2 negative, Ki-67 low); luminal B-like, HER2-negative tumors (ER positive, HER2 negative, Ki-67 high and/or PgR low or negative); luminal B-like, HER2 positive tumors (ER positive, HER2 overexpressed or amplified); HER2 positive, nonluminal tumors (HER2 overexpressed or amplified, ER and PgR negative); triple negative tumors (ER, PgR, and HER2 negative)

$C R$ complete response, $P R$ partial response, $S D$ stable disease, $P D$ progressive disease, $N A$ not assessable, $E R$ estrogen receptor, $P R$ progesterone receptor

largest published retrospective experience with ECT in BC, showed a CR response rate of $40 \%$ as the most favorable outcome among elderly patients. ${ }^{22}$ Consistent with a recent meta-analysis, in the present study the response to treatment in small tumors $(<3 \mathrm{~cm})$ was higher, similar to that seen in ER-positive, low-proliferating tumors (representing the luminal A-like BC subtype) and in patients without ulcerated lesions or visceral metastases. ${ }^{24}$

In particular, the $\mathrm{CR}$ rate in the luminal A-like $\mathrm{BC}$ subtype was $73.9 \%$, which was significantly higher than in triple-negative and $H E R 2$ positive BC patients (57.1 and $54.5 \%$, respectively), independent of tumor size. However, although ECT in triple-negative BC in our series was used after failure of several lines of treatment and in conditioning a highly refractory disease, the $\mathrm{CR}$ rate in this group nonetheless exceeded $50 \%$.

We are aware that clinical evaluation may be a subjective assessment of tumor response. A pilot study including 11 patients with chest wall recurrence from $\mathrm{BC}$ investigated the role of ${ }^{18} \mathrm{~F}$-fludeoxyglucose positron emission tomography (FDG-PET). This study indicates that not only FDG-PET/computed tomography (CT) but also dual time point imaging FDG-PET/CT is promising for evaluation and planning of ECT and could be useful for other localized anticancer treatments as well. ${ }^{25}$ On the other hand, this imaging technique, which is not widely available and which has nonnegligible costs, has a low sensitivity for small tumor deposits, limiting its application in cutaneous oncology. ${ }^{26}$

In our patients, data on local control indicated a 1-year LPFS of $86.2 \%$ within the ECT field (Fig. 2), increasing to $96.4 \%$ in those with CR. In our experience, small $(<3 \mathrm{~cm})$ tumor size represented the main predictor of local control compared to large $(\geq 3 \mathrm{~cm})$ tumor size $(97.4$ vs. $75.6 \%$ at 1 year, respectively).

Skin involvement represents a less frequent but not uncommon event in the metastatic pattern of $\mathrm{BC}$, accounting for $5-30 \%$ of advanced cases in different series. ${ }^{1,2}$ In
FIG. 2 Tumor control after ECT. Kaplan-Meier curves for LPFS in a whole cohort and $\mathbf{b}$ subgroups of patients with lesions $<3 \mathrm{~cm}$ (blue line) and $\geq 3 \mathrm{~cm}$ (yellow line)
A

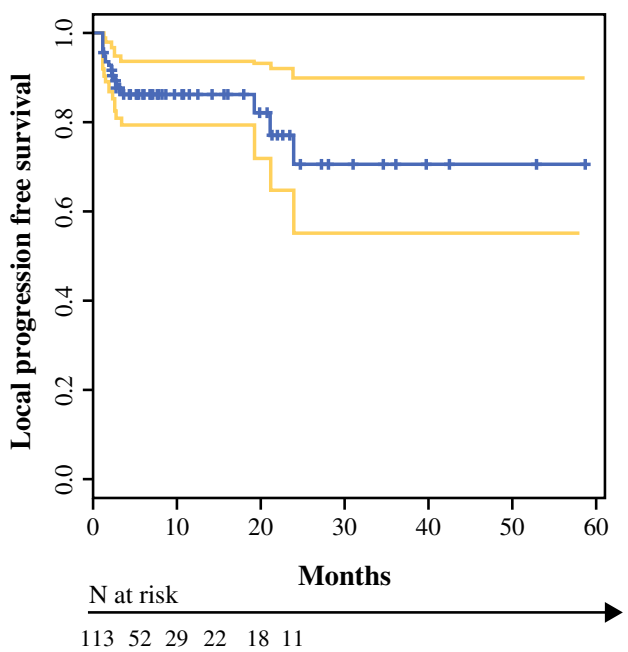

B

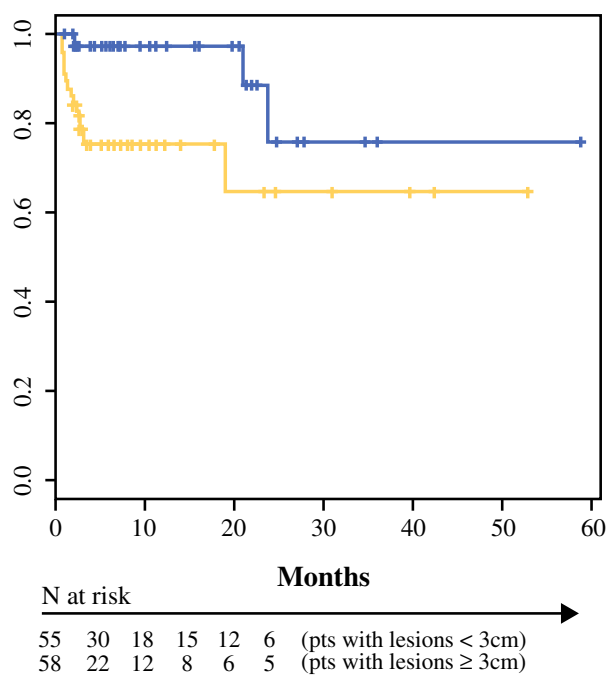


addition to their association with unfavorable prognosis, skin metastases cause strong psychologic distress. ${ }^{16}$ Surgical resection with a radical intent can only be offered to a limited number of patients as a result of multifocality and clinically occult lymphangitic spread. ${ }^{27}$ In these cases, radiotherapy is generally the best option, but it is often unfeasible on previously irradiated tissues and on lesions that have spread on a wide area. Lack of capillary distribution of radiologic facilities in the territory and the long duration of the entire cycle on multiple sessions may represent further criticisms. Conversely, ECT is applicable on preirradiated areas with the possibility to treat many lesions in a single session, without systemic side effects and a favorable toxicity profile. At any rate, ECT is repeatable and can even be performed in an outpatient setting.

Undoubtedly, our findings need broader and prospective confirmation. Furthermore, it will be necessary to clarify whether delaying progression of cutaneous metastases by ECT may provide clinically meaningful benefit to patients, such as delay of disease-related symptoms or preservation of quality of life. In general, the value of progression-free survival, as a surrogate marker for patient benefit, has recently been subjected to critical reappraisal. ${ }^{28}$ In fact, patient-centered outcomes will a crucial issue in future studies on ECT. ${ }^{29,30}$

ACKNOWLEDGMENT We are indebted to Francesca de Terlizzi for her fundamental help with the statistical analysis; we also thank Luigi Corti and Gaetano Castiglione for patient care and Christina Drace for editorial help.

DISCLOSURE The authors declare no conflict of interest.

OPEN ACCESS This article is distributed under the terms of the Creative Commons Attribution 4.0 International License (http:// creativecommons.org/licenses/by/4.0/), which permits unrestricted use, distribution, and reproduction in any medium, provided you give appropriate credit to the original author(s) and the source, provide a link to the Creative Commons license, and indicate if changes were made.

\section{REFERENCES}

1. Zagar TM, Higgins KA, Miles EF, et al. Durable palliation of breast cancer chest wall recurrence with radiation therapy, hyperthermia, and chemotherapy. Radiother Oncol. 2010;97:535-40.

2. Oldenborg S, Van Os RM, Van Rij CM, et al. Elective re-irradiation and hyperthermia following resection of persistent locoregional recurrent breast cancer: a retrospective study. Int $J$ Hyperthermia. 2010;26:136-44.

3. Guarneri V, Conte P. Metastatic breast cancer: therapeutic options according to molecular subtypes and prior adjuvant therapy. Oncologist. 2009;14:645-56.

4. Leonard R, Hardy J, van Tienhoven G, et al. Randomized, double-blind, placebo-controlled, multicenter trial of $6 \%$ miltefosine solution, a topical chemotherapy in cutaneous metastases from breast cancer. J Clin Oncol. 2001;21:4150-9.

5. Mir LM, Orlowski S. Mechanisms of electrochemotherapy. Adv Drug Deliv Rev. 1999;35:107-18.

6. Marty M, Sersa G, Garbay JR, et al. Electrochemotherapy-an easy, highly effective and safe treatment of cutaneous and subcutaneous metastases: results of ESOPE (European Standard Operating Procedures of Electrochemotherapy) study. Eur $J$ Cancer. 2006;4(suppl 11):3-13.

7. Spratt DE, Gordon Spratt EA, Wu S, et al. Efficacy of skindirected therapy for cutaneous metastases from advanced cancer: a meta-analysis. J Clin Oncol. 2014;32:3144-55.

8. Sersa G, Cufer T, Paulin SM, et al. Electrochemotherapy of chest wall breast cancer recurrence. Cancer Treat Rev. 2012;38:37986.

9. Hammond ME, Hayes DF, Dowsett M, et al. American Society of Clinical Oncology/College of American Pathologists guideline recommendations for immunohistochemical testing of estrogen and progesterone receptors in breast cancer. J Clin Oncol. 2010;28:2784-95.

10. Cheang MC, Chia SK, Voduc D, et al. Ki67 index, HER2 status, and prognosis of patients with luminal B breast cancer. $J$ Natl Cancer Inst. 2009;101:736-50.

11. Goldhirsh A, Winer EP, Coates AS, et al. Personalising the treatment of women with early breast cancer: highlights of the St Gallen International Expert Consensus on the primary therapy of early breast cancer. Ann Oncol. 2013;24:2206-23.

12. Mir LM, Gehl J, Sersa G, et al. Standard operating procedures of the electrochemotherapy: instructions for the use of bleomycin or cisplatin administered either systemically or locally and electric pulses delivered by the Cliniporator ${ }^{\mathrm{TM}}$ by means of invasive or non-invasive electrodes. Eur J Cancer. 2006;4(suppl 11):14-25.

13. Eisenhauer EA, Therasse P, Bogaerts LH, et al. New response evaluation criteria in solid tumors: revised RECIST guideline (version 1.1). Eur J Cancer. 2009;45:228-47.

14. US Department of Health and Human Services. NIH-NCI. Common Terminology Criteria for Adverse Events (CTCAE). Version 4.0. May 28, 2009. http://evs.nci.nih.gov/ftp1/CTCAE/ CTCAE.

15. McCaffery M, Pasero C. Pain: clinical manual. 2nd ed. St. Louis: CV Mosby; 1999.

16. Campana LG, Mocellin S, Basso M, et al. Bleomycin-based electrochemotherapy: clinical outcome from a single institution's experience with 52 patients. Ann Surg Oncol. 2009;16:191-9.

17. Campana LG, Valpione S, Falci C, et al. The activity and safety of electrochemotherapy in persistent chest wall recurrence from breast cancer after mastectomy: a phase-II study. Breast Cancer Res Treat. 2012;134:1169-78.

18. Quaglino P, Matthiessen LW, Curatolo P, et al. Predicting patients at risk for pain associated with electrochemotherapy. Acta Oncol. 2015;16:1-9.

19. Larkin JO, Collins CG, Aarons S, et al. Electrochemotherapy. Aspects of preclinical development and early clinical experience. Ann Surg. 2007;245:469-79.

20. Matthiessen LW, Johannesen HH, Hendel HW, et al. Electrochemotherapy for large cutaneous recurrence of breast cancer: a phase II clinical trial. Acta Oncol. 2012;51:713-21.

21. Benevento R, Santoriello A, Perna G, et al. Electrochemotherapy of cutaneous metastases from breast cancer in elderly patients: a preliminary report. BMC Surg. 2012;12(suppl 1):S6.

22. Campana LG, Galuppo S, Valpione S, et al. Bleomycin electrochemotherapy in elderly metastatic breast cancer patients: clinical outcome and management considerations. J Cancer Res Clin Oncol. 2014;140:1557-65. 
23. Mali B, Jarm T, Snoj M, et al. Antitumor effectiveness of electrochemotherpay: a systematic review and meta-analysis. Eur $J$ Surg Oncol. 2013;39:4-16.

24. Mali B, Miklavcic D, Campana LG, et al. Tumor size and effectivness of electrochemotherapy. Radiol Oncol. 2013;47: 32-41.

25. Matthiesen LW, Johannesen HH, Skougaard K, et al. Dual time point imaging fluorine-18 fluorodeoxyglucose positron emission tomography for evaluation of large loco-regional recurrences of breast cancer treated with electrochemotherapy. Radiol Oncol. 2013;47:358-65.

26. Evangelista L, Mezzato C, Felloni G, et al. Current and future perspectives in diagnostic imaging as a guide to targeted/local therapies in breast cancer recurrence. $Q \mathrm{~J} \mathrm{Nucl} \mathrm{Med} \mathrm{Mol} \mathrm{Imaging.}$ 2013;57:367-80.
27. Chagpar A, Meric-Bernstam F, Hunt KK, et al. Chest wall recurrence after mastectomy does not always portend a dismal outcome. Ann Surg Oncol. 2003;10:628-34.

28. Robinson AG, Booth CM, Eisenhauer EA. Disease-free survival as an end-point in the treatment of solid tumours-perspectives from clinical trials and clinical practice. Eur $J$ Cancer. 2014;50:2298-302.

29. Siena S, Peeters M, Van Cutsem E, et al. Association of progression-free survival with patient-reported outcomes and survival: results from a randomised phase 3 trial of panitumumab. Br J Cancer. 2007;97:1469-74.

30. Parikh RB, Kirch RA, Smith TJ, et al. Early specialty palliative care: translating data in oncology into practice. $N$ Engl $J$ Med. 2013;369:2347-51. 